\title{
Prevalencia y detección por PCR anidada de Anaplasma marginale en bovinos y garrapatas en la zona central del Litoral ecuatoriano
}

\section{Prevalence and detection by nested PCR of Anaplasma marginale in cattle and tick in the center of the coast of Ecuador}

${ }^{\bullet}$ Ariel Escobar ${ }^{1}$, Orly Cevallos ${ }^{2}$, Patricio Villarreal ${ }^{1}$, Mercedes Carranza $^{3}$, Helen Carranza ${ }^{3}$, Edgar Pinargote ${ }^{2}$

${ }^{1}$ Escuela de Biología, Facultad de Ciencias Naturales, Universidad de Guayaquil, Ecuador.

•telarieles@hotmail.com; p.villarreal@yahoo.com

${ }^{2}$ Facultad de Ciencias Pecuarias, Campus Finca Experimental "La Maria” km 7 vía Quevedo-El Empalme.

Universidad Técnica Estatal de Quevedo-(UTEQ) EC.120501. cevallos@uteq.edu.ec; edgarpinargote@yahoo.com

${ }^{3}$ Facultad de Ciencias Ambientales, Universidad Técnica Estatal de Quevedo. Campus Ing. Manuel Haz Álvarez, km 1.5

vía a Santo Domingo de los Tsáchilas.EC.120501.Quevedo,Ecuador. mcarranza@uteq.edu.ec; cp_helen@hotmail.com

Resumen

$\mathrm{L}$ a bacteria que provoca la anaplasmosis en bovinos se conoce como Anaplasma marginale, es potencialmente transmitida de forma biológica por garrapatas, moscas y fómites o mediante sangre infectada como consecuencia del uso incorrecto de herramientas quirúrgicas. Hasta la fecha, Ecuador carece de estudios actualizados sobre procedimientos eficientes para el diagnóstico específico y, erradicación de $A$. marginale a través del control de estos insectos, por lo que resulta de gran importancia desarrollar e implementar trabajos relacionados con el uso de esta herramienta molecular, para la detección de la bacteria en vectores de transmisión que provocan la enfermedad en bovinos. En este trabajo, se extrajo ADN eficazmente con el método de Salting Out. Un total de 255 muestras fueron analizadas por PCR anidada, distribuidas del siguiente modo|108| Riphicephalus (Boophilus) microplus, $|85|$ bovinos, de estos el $13.46 \%$ y $85.48 \%$ dieron positivas para la rickettsia, las muestras de Amblyomma spp. $|62|$ todas fueron negativas. El índice de concordancia Kappa |total de bovinos infestados frente a Riphicephalus (Boophilus) microplus|, no fue significativo (0.28). Subsiguientemente, se determinó por $\chi^{2}(\mathrm{p}=0.66)$ que la presencia o ausencia de la enfermedad es independiente del lugar de donde proviene el bovino.

Palabras clave: detección, bovinos, fincas, Salting Out.

\begin{abstract}
$T$ he bacterium causing anaplasmosis in cattle is known as Anaplasma marginale. It is mainly transmitted biologically by ticks, flies, and fomites; it can also be transmitted through infected blood as a result of improper use of surgical tools. Up to this moment, Ecuador lacks updated studies on efficient procedures for specific A. marginale diagnosis and eradication through the control of these insects, therefore it is necessary to develop and implement proposals related to the use of this molecular tool for potential vectors of transmission of this disease in cattle. For this work, DNA was extracted efficiently by the method of Salting Out. A total of 255 samples were analyzed by Nested PCR, distributed as follows |108| Rhipicephalus (Boophilus) microplus, $|85|$ bovine, from these, the $13.46 \%$ and $85.48 \%$ were positive for rickettsia, the samples of Amblyomma spp |62| all were negative. The total Kappa index of infested cattle against Rhipicephalus (Boophilus) microplus|, was not significant (0.28). Subsequently, the presence or absence of disease which was determined by $\chi^{2}(p=0.66)$ does not depend on the location from where the cattle comes.
\end{abstract}

Key words: detection, cattle, farms, Salting Out.

Recibido: 19-noviembre-2014. Recibido en forma corregida: 3-diciembre-2014. Aceptado: 18-marzo-2015. 


\section{Introducción}

A naplasma marginale es una bacteria ampliamente estudiada, varios reportes han determinado el análisis completo de su estructura genómica (Alleman et al., 1993; Kocan et al., 2008). Esta rickettsia pertenece al genogrupo II de las Ehrlichias, siendo además un parasito de los eritrocitos maduros del ganado bovino (Corona y Martínez, 2011). Varios reportes han demostrado que la secuencia genómica del complejo principal de superficie MSP4 es conservada entre aislados de $A$. marginale (De la Fuente et al., 2002, 2009) y, codifica para un sólo gen (Jiménez et al., 2012), por lo que se considera un candidato específico para el diagnóstico de la enfermedad.

Dentro del grupo de la superfamilia Ixodidae se encuentran las garrapatas o ácaros grandes (2$30 \mathrm{~mm}$ de tamaño). En etapa post-embriónica se tornan ectoparásitos hematófagos obligados (Dumler et al., 2001). Las incidencias de las garrapatas en la salud humana $\mathrm{y}$, animal son múltiples, pudiendo causar lesiones severas en sus huéspedes: dermatosis, otoacariasis, además de ser capaces de producir toxinas que provocan la parálisis, incluso la muerte de su hospedero (Guglielmone et al., 2004, 2006). Se debe resaltar asimismo que estos parásitos, son reconocidos como potenciales vectores de bacterias, nematodos, protozoarios, rickettsias y virus (Hoogstraal, 1985; Eriks et al., 1989, 1993; De la Fuente et al., 2009).

Riphicephalus (Boophilus) microplus es conocida como la garrapata del ganado (FAO, 1984; Bock et al., 2008; Kocan et al., 2008) al mismo tiempo, es considerada la más importante a nivel mundial por infectar preferentemente a este tipo de animales (Walker et al., 2003; Estrada et al., 2006). Según reportes, en Ecuador esta garrapata se la relaciona como uno de los vectores responsables de provocar la enfermedad (Sotomayor, 1995; Guglielmone et al., 2004, 2006).

A nivel mundial se ha reportado que existen alrededor de 106 especies de Amblyomma spp., de las cuales 57 se encuentran en la región neotropical (Guglielmone et al., 2004). Según registros, en Ecuador se han descrito varias especies; Amblyomma maculatum (Koch, 1844), A. hirtum (Neuman, 1906), A. darwini (Robinson, 1926), A. williamsi (Schulze, 1936), A. calcaratum y A. incisum (Jones et al., 1972), A. macfarlandi, A. pilosum, A. usingeri (Keirans et al., 1973) y, A. humerale (Barros-Battesti et al., 2002), A. triste (Guglielmone et al., 2004, 2006) entre otras. Este grupo de garrapatas son consideradas altamente perjudiciales para la salud del ganado, ya que producen grandes heridas y facilitan, la entrada de patógenos, los mismos que provocan abscesos, conduciendo a la inflamación en las ubres de las vacas y, afectando drasticamentre el rendimiento en la producción de leche (The Center for Food Security \& Public Healt, 2007).

Este trabajo se llevó a cabo en el área central del Litoral ecuatoriano, con el objetivo de detectar molecularmente y determinar la incidencia de Anaplasma marginale en garrapatas (Riphicephalus (Boophilus) microplus, Amblyomma spp. y, bovinos. Teniendo en cuenta las potencialidades de la técnica de PCR anidada |nPCR| (De la Fuente et al., 2003, 2009; Corona y Martínez, 2011; Corona et al., 2012); se establece que ésta herramienta puede ser utilizada en el diagnóstico de la enfermedad, ya que por su alta sensibilidad y especificidad permite comprobar la presencia de la rickettsia en muestras de tejido sanguíneo y tisular de bovinos y garrapatas respectivamente (Corona y Martínez, 2011; Rojas et al., 2013). Por otro lado, este trabajo proporciona información concerniente para establecer un andén en la vigilancia epidemiológica, monitoreo de posibles vectores resistentes, aplicación de metodologías sustentables de control y diagnóstico, primordiales en la lucha contra esta enfermedad que afecta dramáticamente la salud del ganado vacuno de esta zona.

\section{Materiales y métodos}

\section{Área de recolección de muestras}

Utilizando el método aleatorio simple, se colectaron los especímenes y, sangre a partir de bovinos contaminados. Las muestras procedían de cinco sitios, ubicados en la franja periurbana al cantón Quevedo, provincia de Los Ríos, Ecuador, Haciendas María Verónica y Silvia María, ubicadas en las parroquias La Esperanza y San Carlos, respectivamente. Fincas Dolores María, situada en la parroquia San Cristóbal y Experimental La María perteneciente a la Facultad de Ciencias Pecuarias de la Universidad Técnica Estatal de Quevedo (UTEQ) y, la Empresa Municipal del Camal del cantón Quevedo (EMCQ).

\section{Material animal}

Se colecto aleatoriamente un total de 255 muestras clasificadas en: 170 garrapatas y 85 muestras de sangre de bovinos. Las garrapatas se extrajeron de la ubre, entrepierna y debajo de la cola, posteriormente fueron ubicados en tubos estériles (Eppendorf) que contenía etanol y éter etílico en relación 1:3 respectivamente. Para clasificar e identificar las garrapatas se utilizó un estéreo microscopio (SMZ-140-Motic ${ }^{\circledR}$ ) y se aplicó las claves establecidas por Madder (2010), previo a su almacenamiento a $4^{\circ} \mathrm{C}$ se lavó con agua destilada desionizada y, se fijaron con alcohol etílico al $96 \%$ (Sigma).

Para obtener las muestras de sangre de los bovinos se 
realizaron punciones en la vena yugular o coccígea utilizando agujas (BD Vacutainer®Eclipse ${ }^{\mathrm{TM}}$ ) y, tubos con anticoagulante (EDTA K2-Vacutainer ${ }^{\circledR}$ ), los mismos fueron rotulados y almacenados a $-20^{\circ} \mathrm{C}$. Todas las muestras se trasladaron al laboratorio de Biotecnología (UTEQ).

\section{Purificación y cuantificación del ADN}

Toda la manipulación de los reactivos y, tejidos se realizó en una cámara de flujo laminar (MIN60-C4®), utilizando puntas (epTIPS Box- Eppendorf) y pipetas resistentes al efecto aerosol. Se ensayaron dos protocolos de extracción, Miller et al. (1988) denominado "Salting-Out" y TENS [Tris-Cl pH7.5, 10 $\mathrm{mM}$, ácido etildiaminotetraacético (EDTA), $125 \mathrm{Mm}$ $\mathrm{NaCl}, 1.0 \%$ dodecilsulfato sódico (SDS)] reportado por Wilmer y Barratt (1996). La calidad y concentración de ADN se determinó por fluorometría (Qubit ${ }^{\mathrm{TM}_{-}}$) y Quant-iT ${ }^{\mathrm{TM}}$ dsADN HS Assay Kit (Invitrogen ${ }^{\mathrm{TM}}$ ) respectivamente. Como fuente de $\mathrm{ADN}$ positivo para la detección de $A$. marginale, se utilizó una muestra genómica de la bacteria, concedida por el equipo de la Escuela Politécnica del Ejército (ESPE)-Ecuador.

\section{Amplificación del gen msp4 por PCR anidada}

La nPCR fue optimizada para detectar la rickettsia. Se diseñaron dos juegos de oligonucleótidos basados sobre las secuencias reportadas con el código de acceso GenBank No KM624517.1de la región interna del gen msp4 de Anaplasma marginale. Para la PCR1 los oligonucleótidos fueron |5'CACCATGAATTACAGAGAATTG3' y $\mid 5^{\prime}$ GCTGAACAGGAATCTTGCTCC 3'| que amplifican un fragmento de 846-pb. Para la nPCR los oligonucleótidos $\quad 5^{\prime}$ CTCATCAAATAGCCCGTGGT 3'| y |5' GACGTGCTGCACACAGATTT 3'| amplifican un fragmentos de 200-pb, todos los oligonucleótidos fueron sintetizados por Integrated DNA Technologies, Inc.

La preparación de los reactivos se realizó en una cámara de flujo laminar (MIN60-PCR C4®). Para la PCR1 y PCR2 la mezcla de reactivos fue de $25 \mu \mathrm{L}$ por reacción y contenía $1 \mathrm{X}$ (GoTaq ${ }^{\circledR}$ Flexi DNAPolymerasePromega), $200 \mu \mathrm{M}$ de los dNTPs (Invitrogen ${ }^{\mathrm{TM}}$ ), $10 \mu \mathrm{M}$ de cada uno de los oligonucleótidos, $1.5 \mathrm{mM}$ [MgCl2]2, $0.2 \mu \mathrm{L}$ de Taq polimerasa (Promega) y $19.05 \mu \mathrm{L}$ de agua (UltraPure ${ }^{\mathrm{TM}}$ DNase/RNase-Free DistilledWaterInvitrogen $^{\mathrm{TM}}$ ) y $100 \mathrm{ng}$ de $\mathrm{ADN}$. Las reacciones se desarrollaron en un termociclador (Techne Genius Thermocycler-Model TGENO2TP). Para los ensayos de PCR1 y PCR2 se usó el siguiente programa de amplificación: PCR1 un ciclo a $94{ }^{\circ} \mathrm{C}$ por 5 minutos; 40 ciclos con segmentos de $94{ }^{\circ} \mathrm{C}$ por 1 minuto; 45 segundos a $54{ }^{\circ} \mathrm{C}$ y $72{ }^{\circ} \mathrm{C}$ por un minuto, la extensión final fue a $72{ }^{\circ} \mathrm{C}$ por 7 minutos. PCR2 un ciclo a 94 ${ }^{\circ} \mathrm{C}$ por 3 minutos; 40 ciclos con segmentos de $94{ }^{\circ} \mathrm{C} \mathrm{y}$ $54^{\circ} \mathrm{C}$ por 45 segundos y, $72^{\circ} \mathrm{C}$ por un minuto con una extensión final de $72{ }^{\circ} \mathrm{C}$ por 7 minutos. Los resultados de las amplificaciones fueron depositados en geles de agarosa (Invitrogen ${ }^{\mathrm{TM}}$ ) al 2\% con TAE 1X y, Bromuro de Etidio [0.5 $\mu \mathrm{g} . \mathrm{mL}^{-1}$ ] (Invitrogen ${ }^{\mathrm{TM}}$ ), cada producto de ADN se mezcló con $3 \mu \mathrm{L}$ de tampón de depósito $1 \mathrm{X}$ (BlueJuice $^{\mathrm{TM}}$ Gel Loading Buffer -Invitrogen ${ }^{\mathrm{TM}}$ ), se usó un marcador de $100 \mathrm{pb}$ (Gilco BRL), y partieron por 35 minutos a $75 \mathrm{~V}$, finalmente los productos se visualizaron en el documentador de geles E-Gel ${ }^{\circledR}$ ImagerSystem (Life Technologies ${ }^{\mathrm{TM}}$ ).

\section{Diseño estadístico}

Para la extracción, purificación y cuantificación del ADN se usó un diseño factorial de seis tratamientos con cinco repeticiones y tres lecturas, el análisis de varianza (ANOVA) se determinó con el método de Tukey $(p<0.05)$. Finalmente para establecer el mejor tratamiento se aplicó una estimación paramétrica bilateral a través de intervalos de confianza.

En cuanto a la optimización de la nPCR se usó un diseño factorial de seis tratamientos con cinco repeticiones, con una ANOVA $(\mathrm{p}<0.05)$ no paramétrica por medio del método de Kruskal Wallis y, para los datos no categorizados se utilizó la prueba $\chi^{2}$.

Para determinar el grado de concordancia entre las muestras infectadas de sangre $\mathrm{y}$, las garrapatas portadoras de la rickettsia obtenidas de un mismo bovino, se utilizó la prueba de Kappa. Los datos fueron analizados con el paquete estadístico GraphPadPrism 2.0 (Graphpad Software Inc., San Diego, CA).

\section{Resultados y discusión}

T os datos sobre la purificación y cuantificación genómica fueron sometidos a la aplicación de varianza; en cuanto al modelo, método, fuente $\mathrm{y}$ la interacción entre método/fuente $[|\mathrm{p}=0.001|$, $|p=1.11 \times 10-16|, \quad|p=1.04 \times 10-14| \quad$ y $\quad|p=5 \times 10-7|]$ respectivamente; para complementar y robustecer esta información, se sometió a las pruebas de intervalos de confianza calculados. Los resultados demuestran que el tratamiento con el método de Salting Out generó una mayor cantidad y, calidad de ADN en todas las muestras de garrapatas, no se examinó estadio evolutivo de los ácaros, las mismas que oscila entre 3980.63 a 5275.00 ng $\mathrm{mL}^{-1}$, estos resultados concuerdan con los reportados por Oslinger et al. (2006); Piedrahíta et al. (2008) y Escobar et al. (2014), quienes aplicaron este método para extraer eficientemente ácidos nucleicos $|\mathrm{AN}|$. Por otro lado, el método de TENS sobre el tejido sanguíneo 
produjo un ADN entre 1667.7 a $1962.98 \mathrm{ng} \mathrm{mL}^{-1}$, según reportes de Zhou et al. (1990) mencionan que este método no es muy eficiente para la extracción genómica en tejido animal (Cuadro 1).

Cuadro 1. Intervalos de Confianza de concentraciones de ADN extraídos en $\mathrm{ng} \mathrm{mL}^{-1}$

\begin{tabular}{llccc}
\hline Método & \multicolumn{1}{c}{ Fuente } & $\mathrm{n}$ & $\mathrm{Li}$ & $\mathrm{Ls}$ \\
\hline SO & Amblyomma spp. & 15 & 3980.63 & 5275.37 \\
SO & Boophilus spp. & 15 & 5539.64 & 5887.03 \\
SO & Tejido sanguíneo & 15 & 2280.55 & 3500.78 \\
TENS & Amblyomma spp. & 15 & 3161.24 & 4042.76 \\
TENS & Boophilus spp. & 15 & 2160.29 & 2949.05 \\
TENS & Tejido sanguineo & 15 & 1667.69 & 1962.98 \\
\hline
\end{tabular}

Li: Límite inferior; Ls: Límite superior

La ultrasensibilidad de detección por la PCR anidada permitió determinar qué; del total de las muestras analizadas, el $13.46 \%$ de las garrapatas Riphicephalus (Boophilus) microplus fueron positivas para A. marginale, de estos el $22.58 \%$ de la población pertenecen a las muestra extraídas de las haciendas y fincas; el $77.42 \%$ corresponden a las extraídas de la EMCQ. Mientras tanto que para Amblyomma spp. lespecie no identificada |todas las muestras fueron negativas (Figura 1); varios reportes, entre ellos (Davey y Georgi, 2002; Kocan et al., 2004), determinaron que la inexistencia de A. marginale en Amblyomma spp., se debe a que éste género no es un transmisor de la rikettsia. Por otro lado Corona y Martínez (2011) sugieren que el estado del portador de anaplasmosis, es un fenómeno que no está suficientemente entendido $\mathrm{y}$, es posible que bajo determinados niveles de parasitemia de los animales individuales no sean capaces de trasmitir la bacteria, mencionando además que existen factores externos e internos que pueden ser capaces de alterar los niveles de parasitemia de un individuo. En cuanto a la detección promedio de la rickketsia en la población total de bovinos, fue del $85.48 \%$, lo cual coincide considerablemente con los datos reportados por Rojas et al. (2010) y Kumsa et al. (2014), quienes determinaron que el grado de contaminación por A. marginale en un rebaño puede ser significtivo en parentales, como en su progenie, en las zonas de prevalencia de la enfermedad.

Los datos de amplificación del ADN bacteriano (Figura 1) en ambas muestras |total de bovinos infestados $\mid \mathrm{y}$, Riphicephalus (Boophilus) microplus, fueron comparados con el test Kappa, obteniendo un

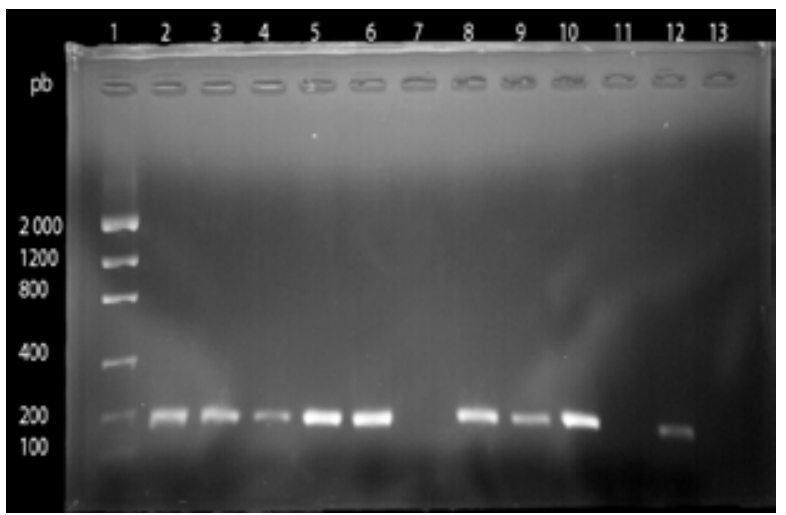

Figura 1. Detección por PCR anidada para $A$. marginale en muestras de ADN. Línea 1: Marcador de peso molecular 100 pb (Gilco BRL), 2-3-4: Muestras de Riphicephalus (Boophilus) microplus, 5-6-12: Muestras de bovinos, 7: Control de extracción de ADN (garrapatas), 8-9: ADN de $A$. marginale (ESPE), 10: ADN de A. marginale (UTEQ), 11: Control de agua (PCR), 13: Control de extracción de ADN (bovinos)

índice de concordancia de 0.28703 , de acuerdo a la escala de referencia de la prueba, es una correlación baja, según varios reportes (Scoles et al., 2008; Aubry y Geal, 2011; Kumsa et al., 2014) sugieren que no existe una relación directa entre la garrapata, su hospedero y el grado de contaminación de la bacteria, además, otros autores determinan que la habilidad de la garrapata en calidad de vector para adquirir A. marginale de un animal infectado, es medida estrictamente por el nivel de la parasitemia del mismo durante su alimentación (Futse et al., 2003), por lo que no todas las garrapatas ingurgitadas pueden quedar infectadas (Ribeiro y Lima, 1996; De la Fuente et al., 2002; Kumsa et al., 2014).

El mayor número de bovinos infestados por garrapatas fueron de la EMCQ, los mismos que procedieron de varios sitios; tanto dentro y, fuera de la provincia. De acuerdo a la locación de origen y, los análisis por la n-PCR de las muestras positivas para anaplasmosis, se determinó que la mayor cantidad de animales provienen de la provincia de Santo Domingo de los Tsáchilas con el 88\% (30/34); Cotopaxi 100\% (10/10); Los Ríos 90\% (9/10); Guayas 100\% (5/5); Esmeraldas $83 \%(5 / 6)$ y bovinos sin registro de procedencia el $85.70 \%(6 / 7)$, similares hallazgos fueron reportados por Escobar et al. (2014), los mismos que determinaron una alta prevalencia enzoótica en estos sitios, reforzando así una fuerte correlación con los datos obtenidos en este trabajo. Utilizando la prueba de $\chi^{2} \mathrm{y}$, de acuerdo a la plataforma de la evidencia muestral recogida, se determinó que la presencia o ausencia de la enfermedad es independiente del lugar de donde proviene el bovino $(\mathrm{p}=0.6625)$. 


\section{Conclusiones}

E protocolo para la extracción de ADN genómico Salting Out modificado, fue altamente eficiente al usarlo en la detección de $A$. marginale, en tejidos sanguíneo y tisular de bovinos y garrapatas respectivamente. Se determinó por la nPCR que la mayor proporción de infectados por A. marginale fue en bovinos, subsiguientemente en garrapatas Riphicephalus (Boophilus) microplus y, ausencia en Amblyomma spp. El índice de concordancia sobre la detección de la bacteria en garrapatas y bovinos, fue baja, se considera que no existe una relación directa entre la bacteria y los hospederos. Los animales que llegan a la EMCQ frecuentemente provienen de cinco provincias y, la mayor cantidad de bovinos con resultados positivos para la anaplasmosis fueron procedentes de Santo Domingo de los Tsáchilas, además se determinó que la presencia o ausencia de la enfermedad es independiente del lugar de donde provienen.

\section{Bibliografía}

Alleman, A.R., Kamper, S.M., Viseshaku, N. and Barbet, A.F. 1993. Analysis of Anaplasma marginale genome by pulsed-field electrophoresis. Gene Microbiology 139(10): 2439-44

Aubry, P. and Geal, D.W. 2010. A review of Bovine Anaplasmosis. Transboundary and Emerging Diseases. Canadian Food Inspection Agency, Ottawa, ON, Canada 58(1): 1-30.

Barros, B., Jerozolimski, D.M., Onofrio, V.C. and Arzua, M. 2002. Amblyomma humerale Koch, 1844 (Acari: Ixodidae) on tortoise, Geochelon denticulata (L.) and Geochelone carbonaria (Spix) from Kayapó indian land, State of Pará, Northern Brazil.» Abst. 11th Congress Acarol. Mérida 159-160.

Bock, R.E., Jackson, L.A., De Vos, A.J., Jorgensen, W.K. 2008. Babesiosis of Cattle. En: Bowman, A., Nutall, P. (Eds.), Ticks: Biology, Disease and Control. Cambridge University Press 325 p.

Corona, B., Obregón, D., Martínez, S., Espinoza, I., Fernandez, H., Enríquez, R. 2012. Detección por PCR de Anaplasma marginale en búfalos de la región occidental de cuba. Salud Animal 34(1): 11-18.

Corona, B. and Martínez, S. 2011. Detección de Anaplasma marginale en bovinos, mediante la amplificación por PCR del gen msp5. Salud Animal 33(1): 24-3.

Davey, R.B. and Georgi, J.E. 2002. Efficacy of macrocyclic lactone endectocides against
Boophilus microplus (Acari: Ixodidae) infested cattle using different pour-on application treatment regimes. Journal of Medical Entomology 39: 763769.

De la Fuente, J., Van Den Bussche, R.A., Garcia, J.C., Rodríguez, S.D., García, M.A., Guglielmone, A.A., Mangold, A.J., Friche, P.L., Barbosa, R. F., Blouin, E.F., Kocan, K.M. 2002. Phylogeography of New World isolates of Anaplasma marginale based on major surface protein sequences. Veterinary Microbiology 88: 275-285.

De la Fuente, J., Golsteyn, E., Van Den Bussche, R.A., Hamilton, R.G., Tanaka, E.E., Druhan, S.E., and Kocan, K.M. 2003. Characterization of Anaplasma marginale Isolated from North American Bison. Applied and Environmental Microbiology, Aug., p. 5001-5005.

De la Fuente, J., Kocan, K.M., Blouin, E.F., Zivkovic, Z., Naranjo, V., Almazán, C., Esteves, E., Jongejan, J., Daffre, S. and Mangold, A.J. 2009. Functional genomics and evolution of tick-Anaplasma interactions and vaccine development. Veterinary Parasitology in press $175-186$

Dumler, J.S., Barbet, A.C., Bekker, C.P., Dasch, G.A., Palmer, G.H., Ray, S.C., Rikihisa, Y., Rurangirwa, F.R. 2001. Reorganization of the genera in the families Rickettsiaceae and Anaplasmataceae in the order Rickettsiales: unification of some species of Ehrlichia with Anaplasma, Cowdria with Ehrlichia and Ehrlichia with Neorickettsia, descriptions subjective synonyms of Ehrlichia phagocytophila. International Journal of Systematic and Evolutionary Microbiology 51:2145-2165.

Eriks, I.S., Stiller, D., Palmer, G.H. 1993. Impact of persistent Anaplasma marginale rickettsemia on tick infection and transmission. Journal of Clinical Microbiology 31:2091- 2096.

Eriks, I.S., Palmer, G.H., McGuire, T.C., Barbet, A.F. 1989. Detection and quantification of Anaplasma marginale in carrier by using a nucleic acid probe. Journal of Clinical Microbiology 27:279- 284.

Escobar, A., Cevallos, O., Villareal, P., Zambrano, S., Nieto, H., Carranza, M., Pinargote, E. 2014. Prevalencia de anaplasmosis en bovinos de la zona central del Litoral ecuatoriano. Spanish Journal of Rural Development, 5(2): 41-47

Estrada, P.A., Bouattour, A., Camicas, J.L., Guglielmone, A., HorakI, J. F., Latif, A., Pegram, R., Walker, A.R. 2006. The known distribution and ecological preferences of the tick subgenus Boophilus (Acari: Ixodidae) in Africa and Latin America. Experimental and Applied Acarology, 38:219-235. 
FAO. 1984. Tick and Tick Borne Diseases Control: A Practical Field Manual. (Vol. I-II): FAO-UNDP. p. 297: 374-382.

Futse, J.E., Ueti, M.W., Knowless,D.P. and Palmer, G.H. 2003. Transmission of Anaplasma marginale by Boophilus microplus: Retention of vector Competence in the Absence of Vector-Pathogen Interaction. Journal of Clinical Microbiology 41(8): 3829-3824.

Guglielmone, A.A., Bechara, G.H., Szabó, M.P., Barros, D.M., Faccini, J.L., Labruna, M.B., De La Vega, R., Arzua, M., Campos, M., Furlong, J., Mangold, A.J., Martins, J., Rodríguez, M., Venza, J.M., Estrada, P.A. 2004. Garrapatas de importancia médica y veterinaria: América Latina y el Caribe. The Netherlands: International consortium on ticks and tick-borne Diseases (IcttD - 2). 173 p.

Guglielmone, A.A., Beati, L., Barros, B,D., Labruna, M.B., Nava, S., Venzal, J.M., Mangold, A.J., Szabó, M.P., Martins, J.R., González, A.D., Estrada, P. 2006. A Ticks (Ixodidae) on humans in South America. Experimental and Applied Acarology, 40: 83-100.

Hoogstraal, H. 1985. Argasid and nuttalliellid ticks as parasites and vectors. Advances in Parasitology (U.S. Naval Medical Research) 24(2): 135-238.

Jiménez, O., Carlos, A.V., Murguía, S., Nayelli, O., Edmundo, E., Miguel, Á., De la Torre, F., Rosario, C., Domínguez, D., García, D., Rodríguez, C. 2012. Diversidad genética de la región variable de los genes mspla y msp4 en cepas de Anaplasma marginale de México. CEPAS DE Rev M Aenxap Claiesnmca Pmecaur g2in0a112e; 3(3): 373-387.

Jones, E.K., Clifford, C.M., Keirans, J.E. and Kohls, G.M. 1972. The ticks of Venezuela (Acarina: Ixodoidea) with a key os species of Amblyomma in the Western Hemisphere. Brigham Young University science bulletin. Biological series 17: $1-11$.

Keirans, J.E., Hoogstraal, H. and Clifford, C.M. 1973. The Amblyomma (Acarina: Ixodoidea), parasitic on giant tortoise (Reptilia: Testudinae) of the Galapagos Island. Annals of Entomological Society of America 673-688.

Kocan, K.M., De la Fuente, J., Blouin, E.F. 2008. Advances toward understanding the molecular biology of the Anaplasma-tick interface. Front Biosci 13: 7032-7045.

Kocan, K.M., De la Fuente, J., Blouin, E.F. and García, J.C. 2004. Anaplasma marginale (Rickettsiales: Anaplasmataceae): recent advances in defining host-pathogen adaptations of a tick-borne rickettsia. Parasitology 129: 285-300.

Koch, C.L. 1844. Systematische Übersicht über die
Ordnung der Zecken. Archiv für Naturgeschichte 10: 217-239.

Kumsa, B., Manuela, S., Sori, T., Cinzia, T., Reta, D., Dinka, A., Marco, M. and Cassini, R. 2014. Molecular detection of piroplasms in ixodid ticks infesting cattle and sheep in western Oromia, Ethiopia. Trop Anim Health Prod. 46:27-31.

Madder, M., Horak, I., Stoltsz, H. 2010. Ticks: Tick identification. Acceso 13 de enero de 2014. Disponible en http://http://www.afrivip.org/sites/ default/files/identification_complete 1.pdf.

Miller, S.A., Dykes, D.D. and Polesky, H.F. 1988. A simple salting out procedure for extracting DNA from human nucleated cells. Nucleic Acids Reserch (Memorial Blood Center of Minneapolis) 16(3): 1215.

Neuman, L.G. 1906. Notes sur les Ixodidés. IV.» Archives Parasitology 10: 195-219.

Oslinger, A., Muñoz, J.E., Álvarez, L.Á, Ariza, F., Moreno, F., Posso, A. 2006. Caracterización de cerdos criollos colombianos mediante la técnica molecular RAMs. Acta Agronómica (Colombia). 55(4): 45-50.

Piedrahíta, A.M., Posso, A., Álvarez, L.Á. 2008. Variabilidad genética de Hartón del Valle mediante RAM. Acta Agronómica (Palmira). 57(1): 71-76.

Ribeiro, M.F. and Lima, J.D. 1996. Morphology and development of Anaplasma marginale in midgut of engorged female ticks of Boophilus microplus. Veterinary Parasitology 61: 31-39.

Robinson, L.E. 1926. Ticks. A monograph of the Ixodoidea. Part IV. The genus Amblyomma. Cambridge University Press: 302.

Rojas, N.A., Romero, O., Valbuena, H., Crisante, G., Rojas, A. and Añez, N. 2010. Detección de transmisión transplacentaria de Anaplasma marginale en bovinos asintomáticos. Revista Científica, FCV-LUZ / 20(4): 377-382.

Rojas, T., Rueda, A., Díaz, D.M., Esaobo, N., Benavides, J., Imbachi, L., Álvarez, R., López, R. 2013. Identificación de Ehrlichia canis (Donatien \& Lestoquard) Moshkovski mediante PCR anidada. Veterinaria y Zootecnía 7(1): 37-48.

Schulze, P. 1936. The Norwegian zoological expedition to te Galapagos Island. Die Ixodoidea der Galapagos-Inseln. Meddelelser fra det Zoologiske Museum 45: 155-162.

Scoles, G.A., Miller, J.A. and Foil, L.D. 2008. Comparison of the efficiency of biological transmission of Anaplasma marginale (Ricketssiales: Anaplasmataceae) by Dermacentor andersoni Stiles (Acari; Ixodidae) with mechanical transmission by the horse fly, Tabanus fuscicostatus Hine (Diptera: Muscidae). Journal or Medical 
Entomology 45(1): 109-114.

Sotomayor, L., Gonzales, T. 1995. Curso de enfermedades infecciosas de los animales. Guayaquil: Escuela de Medicina Veterinaria de la Universidad de Guayaquil.

The Center for Food Security \& Public Healt. 2007. Amblyomma variegatum \& Amblyomma hebraeum and Rhipicephalus (Boophilus) microplus. Iowa State University of Science and Technology.

Walker, A.R., Bouattour, A. and Preston, P.M. 2003. Ticks of domestic animals in Africa; a guide to identification of species. Rdinburgh: Bioscience Reports.
Wilmer, W.J. and Barratt, E.M. 1996. A non-lethal method of tissue sampling for genetic studies of chiropterans. Bat Res. News 37: 1-3.

Zhou, C., Yang, Y. and Jong, A.Y. 1990. Mini-prep in ten minutes. Biotechniques 8(2): 172-173. 